\title{
Estructura poblacional de Pinus radiata D.Don en fragmentos de Bosque Maulino en Chile central
}

\section{Population structure of Pinus radiata D.Don in fragments of Maulino Forest in Central Chile}

\author{
Persy Gómez ${ }^{1}$, Ramiro Bustamante ${ }^{2,}{ }^{3}$, José San Martín ${ }^{4}$ \& Steffen Hahn ${ }^{1}$ \\ ${ }^{1}$ Jardín Botánico, Universidad de Talca, Casilla 747, Talca, Chile. \\ ${ }^{2}$ Facultad de Ciencias, Universidad de Chile, Casilla 653, Santiago-Chile. \\ ${ }^{3}$ Instituto de Ecología y Biodiversidad (IEB), Facultad de Ciencias, Universidad de Chile, Casilla 653, Santiago, Chile. \\ ${ }^{4}$ Instituto de Biología Vegetal y Biotecnología, Universidad de Talca, Casilla 747, Talca, Chile. \\ jbotanico@utalca.cl
}

\begin{abstract}
In this note we examine the population structure of Pinus radiata, exotic plant that is invading fragments of the Maulino Forest. We evaluate pine density, age, proportion of reproductive individuals across 8 forest fragments. Our results indicate a high proportion of young individuals and reproductive individuals growing inside fragments. Moreover, the fragment size is negatively correlated with pine density but, the proportion of reproductive individuals are not correlated with this landscape attribute. Our result indicate the existence of a pine population that is actively invading forest fragments, a fact that needs to be considered in conservation plans of this native forest.
\end{abstract}

Los bosques nativos de Chile han sufrido una larga historia de perturbaciones humanas. Estas perturbaciones se han intensificado a partir del siglo XX (Donoso \& Lara 1995). Los cambios en el paisaje forestal en la zona central de Chile se incrementaron a partir de 1940, mediante la explotación del bosque nativo, la proliferación de incendios masivos y sobre todo con el incremento de las plantaciones utilizando árboles exóticos, donde destaca Pinus radiata D.Don, una conífera originaria de California, E.U.A. (Donoso 1983, Lara \& Veblen 1993, Ramírez et al. 1984). Estas plantaciones han aumentado sistemáticamente en los últimos años debido a su rentabilidad económica (Hechenleitner et al. 2005).

Debido a las diferentes actividades antrópicas, la superficie y cobertura del Bosque Maulino costero ha declinado significativamente (Donoso \& Lara 1995). Este tipo de bosque, posee una alta diversidad de especies debido a su condición de bosque transicional entre la vegetación mediterránea y los bosques templados del sur de Chile. Posee además elevados grados de endemismos (San Martín \& Donoso 1996). No obstante lo anterior, es uno de los bosques más amenazados de nuestro país (Echeverría et al. 2006). Entre los años 1975 y 2000, la superficie del bosque original se ha reducido en un $67 \%$ y ha sido reemplazado por plantaciones de $P$. radiata (Echeverría et al. 2006), generando un paisaje dominado por plantaciones de pino rodeando fragmentos remanentes de Bosque Maulino.
La fragmentación del bosque nativo y su reemplazo por plantaciones de pino generan un escenario adecuado para que ocurra invasión de $P$. radiata desde las plantaciones, un fenómeno que ha sido documentado en otras regiones del mundo (Richardson \& Brown 1986, Kruger et al. 1989, Richardson \& Higgins 1998). De hecho, las plantaciones forestales, liberan cada año una gran cantidad de semillas hacia el paisaje, lo cual, incrementa la probabilidad de invasión sólo por un efecto masa (Richardson \& Brown 1986, Kruger et al. 1989, Richardson \& Higgins 1998). Una especie es considerada invasora en un nuevo ecosistema cuando es capaz de sobrevivir, establecerse y reproducirse fuera de su hábitat original compitiendo fuertemente con las especies locales (Richardson et al. 2000).

Los individuos de $P$. radiata comienzan a producir semillas a partir de los 5-6 años de edad y continúan siendo reproductivos hasta los 25 años cuando son extraídos por las empresas forestales (Lara \& Veblen 1993). Así, los fragmentos de Bosque Maulino están sometidos a una lluvia de semillas permanente (Bustamante \& Simonetti 2005) y están siendo invadidos por $P$. radiata (Bustamante et al. 2003). En este marco, es relevante examinar las características de las poblaciones de esta especie introducida de modo de inferir la magnitud de este proceso invasivo. El objetivo de esta investigación fue determinar la estructura poblacional de $P$. radiata en ocho fragmentos de Bosque 
Maulino (35-36 $\mathrm{S})$. Específicamente, el estudio se desarrolló en la comuna de Pelluhue, provincia de Cauquenes, Región del Maule, Chile central, y en donde se evaluó la densidad poblacional, la estructura de edades y la proporción de individuos reproductivos; además estas variables se correlacionaron con el tamaño de los fragmentos.

Se trabajó en ocho fragmentos dominados por la especie Nothofagus glauca (Phil.) Krasser, cuyas superficies varían entre las 3 ha y las 152 ha (Fig.1). Se realizaron 162 cuadrículas de 10 x $10 \mathrm{~m}$, distribuidos en los 8 fragmentos (Tabla I). En cada cuadrícula, se contabilizaron todos los individuos de $P$. radiata y se les estimó la edad por conteo de sus anillos de crecimiento (Davel \& Ortega 2003, Donoso et al. 2008). Además, se determinó el estado reproductivo de los individuos observando la presencia/ausencia de conos reproductivos.
Se encontraron 631 individuos de $P$. radiata para los ocho fragmentos de Bosque Maulino (Tabla I), el 69\% de los individuos no sobrepasan los 3 años de edad (Fig.2). Los individuos reproductivos encontrados en los 8 fragmentos fueron en total 69, siendo más frecuentes los individuos entre 10 y 45 años; además se observó que los individuos inician su edad reproductiva a los 6 años.

La densidad de pinos se correlacionó significativa y negativamente con el tamaño de los fragmentos (Rho de Spearman -0,9762, p < 0,001) (Fig.3). Lo mismo ocurrió para la densidad de individuos jóvenes (0-3 años) (Rho de Spearman $-0,8810, \mathrm{p}<0.01)$, no así con los individuos reproductivos de $P$. radiata (Rho de Spearman -0,2619, $\mathrm{p}>$ $0,1)$. En resumen, a mayor tamaño, menor es la probabilidad de encontrar pinos en los fragmentos.

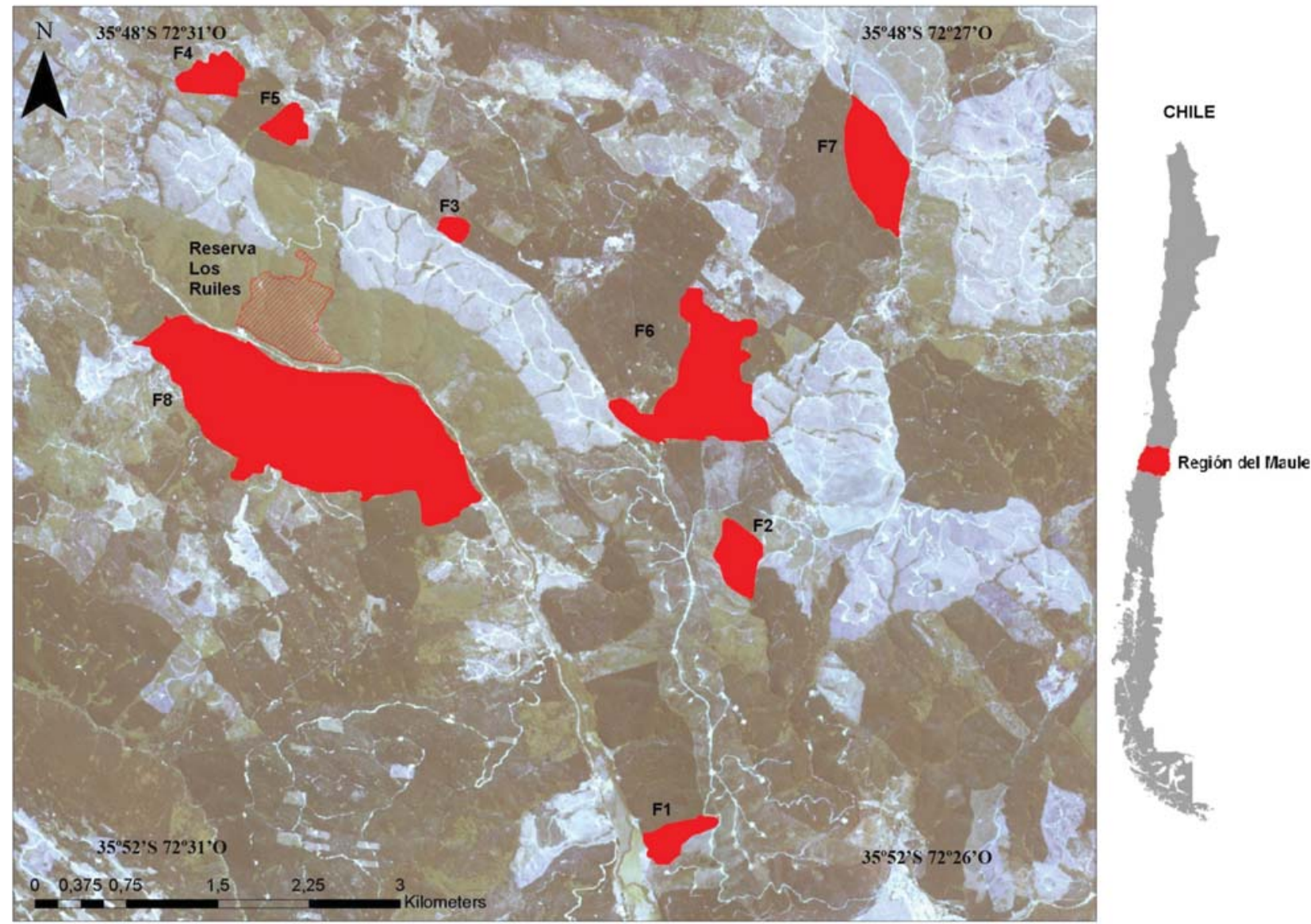

Figura 1. Distribución espacial de los fragmentos de Bosque Maulino, Provincia de Cauquenes, Región del Maule, Chile (F=fragmento).

Figure 1. Spatial distribution of Maulino Forest fragments, Provincia de Cauquenes, Maule Region, Chile (F= fragment). 
Pinus radiata en bosque maulino: GoMEz, P. ET AL.

Tabla I. Tamaño de los fragmentos y densidad de Pinus radiata (Ind/ ha) en ocho fragmentos de Bosque Maulino, Provincia de Cauquenes, Región del Maule, Chile.

Table I. Fragment size and Pinus radiata density (Ind/ ha) across eight fragments of Maulino Forest, Provincia de Cauquenes, Maule Region, Chile.

\begin{tabular}{ccccc}
\hline Fragmento & $\begin{array}{c}\text { Superficie } \\
(\mathrm{ha})\end{array}$ & Superficie muestreada (m²) & Abundancia (Ind/fragmento) & Densidad (Ind/ha) \\
\hline 1 & 11 & 1100 & 42 & 3.8 \\
2 & 19 & 1500 & 61 & 3.2 \\
3 & 3 & 300 & 41 & 2.9 \\
4 & 20 & 1600 & 58 & 4.3 \\
5 & 7 & 700 & 30 & 0.83 \\
6 & 70 & 3500 & 58 & 2.7 \\
8 & 53 & 3000 & 143 & 1.3 \\
\hline Total & 152 & 4500 & 198 & 32.73 \\
\hline
\end{tabular}

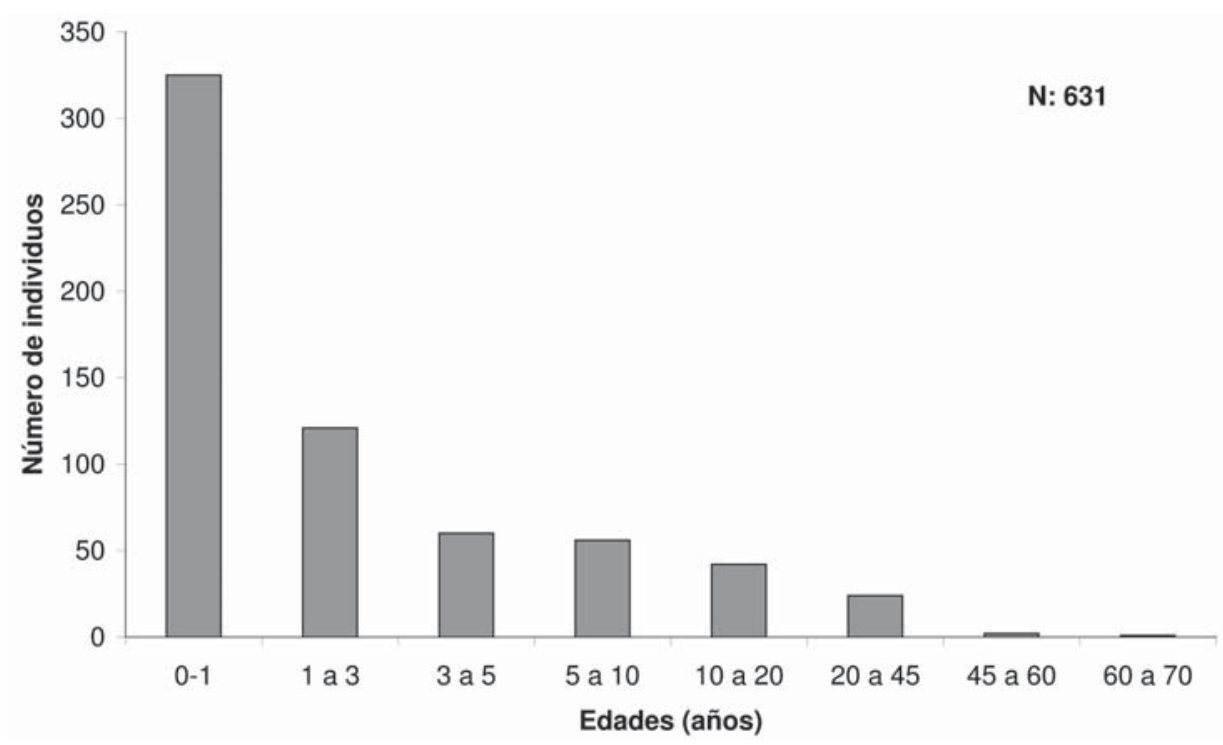

Figura 2. Estructura de edades de Pinus radiata para los ocho fragmentos de Bosque Maulino, Provincia de Cauquenes, Región del Maule, Chile.

Figure 2. Age structure of Pinus radiata for eight fragments of Maulino Forest, Provincia de Cauquenes, Maule Region, Chile. 


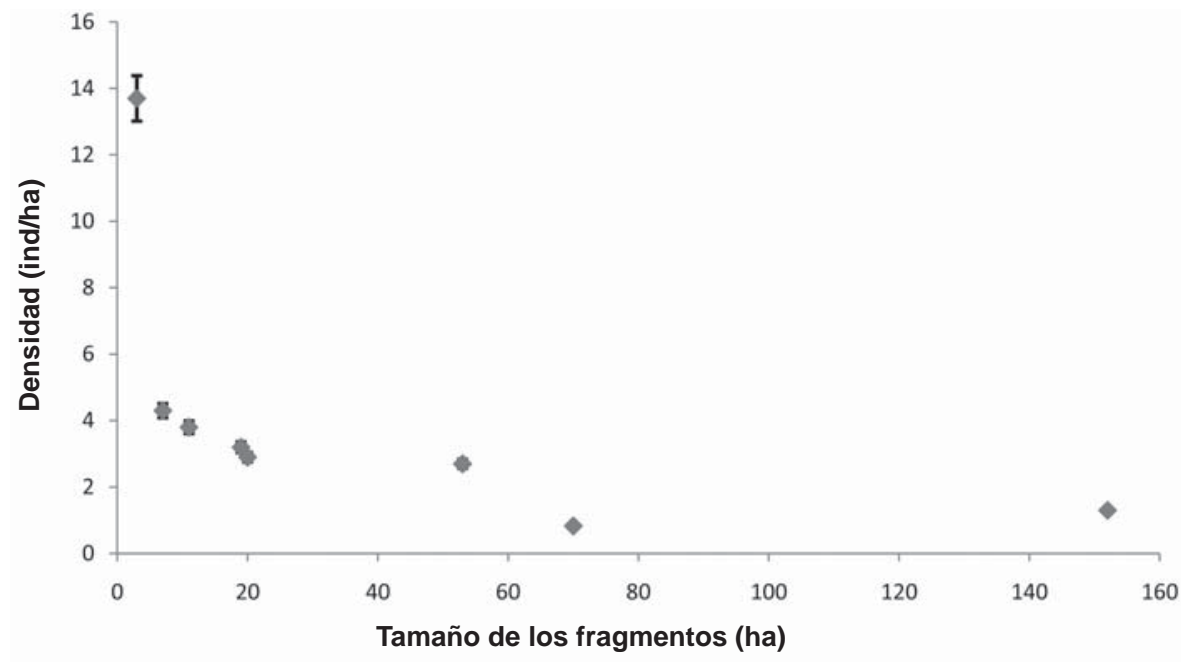

Figura 3. Correlación entre la densidad de Pinus radiata (Ind/ha) y el tamaño de los fragmentos de Bosque Maulino (ha), Provincia de Cauquenes, Región del Maule, Chile (se muestran los promedios \pm 1.96 ee).

Figure 3. Correlation between Pinus radiata density (Ind/ha) and the forest fragment size (ha), Provincia de Cauquenes, Maule Region, Chile (average \pm 1.96 ee).

De estos resultados se concluye que $P$. radiata ha invadido los fragmentos de Bosque Maulino, proceso que aún continúa. Así lo indican la predominancia de individuos jóvenes que no superan los 3 años de edad y la existencia de individuos reproductivos en los fragmentos, característica que le otorga ser catalogada como una especie invasora (Richardson et al. 2000). La escasa densidad de individuos adultos produciendo semillas al interior de los fragmentos de Bosque Maulino, puede ser debido a la tala de estos ejemplares con fines maderables. La presencia de $P$. radiata en los fragmentos de Bosque Maulino puede ser producto de un efecto conjunto de semillas que llegan desde la matriz circundante (plantaciones de pinos), más las producidas por pinos adultos ya establecidos. Los primeros individuos de $P$. radiata habrían colonizado los fragmentos de Bosque Maulino a lo menos el año 1945. El tamaño del fragmento parece ser un buen indicador de la susceptibilidad del Bosque Maulino a ser invadido: fragmentos pequeños son más susceptibles a la invasión mientras que fragmentos grandes sería más resistentes. Considerando que a futuro las plantaciones de pinos continuarán rodeando a los fragmento de Bosque Maulino, se recomienda mantener la complejidad estructural de estos fragmentos para evitar la invasión.

\section{AGRADECIMIENTOS}

Se agradece al proyecto "Estructura de redes mutualistas en bosques fragmentados”. Anillo de Investigación en Ciencia y Tecnología, PBCT, Chile. Se agradece además a Marcela Espinoza por su gran colaboración en terreno.

\section{BIBLIOGRAFÍA}

Bustamante, R.O., I. Serey \& S.T.A. Pickett. 2003. Forest fragmentation, plant regeneration and invasion processes in Central Chile. In: G. Bradshaw, P. Marquet \& M. Mooney (eds.), How landscapes change: Human disturbance and ecosystem fragmentation in the Americas: 145-160. Springer-Verlag, New York, USA.

Bustamante, R.O. \& J.A. Simonetti. 2005. Is Pinus radiata invading the native vegetation in central Chile?, demographic responses in a fragmented forest. Biological Invasions 7: 243-249.

Davel, M. \& A Ortega. 2003. Estimación del índice de sitio para pino oregón a partir de variables ambientales en la Patagonia Andina Argentina. Revista Bosque 24(1): 5569.

Donoso, C. 1983. Árboles nativos de Chile. Guía de reconocimiento. Valdivia, Chile. Alborada. 116 pp.

Donoso, C. \& A. Lara.1995. Utilización de los bosques nativos de Chile: pasado, presente y futuro. En: J.J. Armesto, C. Villagrán \& M.T.K. Arroyo (eds.), Ecología de los Bosques Nativos de Chile. pp. 363-388. Editorial Universitaria, Santiago.

Donoso, C., M.E. González, M. Cortés, C. González, P. Donoso \& M. Hernández. 2008. Poblaciones de araucaria enana (Araucaria araucana) en la Cordillera de Nahuelbuta, Chile. Revista Bosque 29(2): 170-175.

Echeverría, C., A. Newton, A. Lara, JM. Rey-Benayas \& D. Coomes. 2006. Impacts of forest fragmentation on species composition and forest structure in the temperate landscape of southern Chile. Global Ecology and Biogeography 16: 426-439.

Hechenleitner, P., M. Gardner, P. Thomas, C. Echeverría, B. Escobar, B. Brownles \& C. Martínez. 2005. Plantas 
amenazadas del Centro-Sur de Chile. Universidad Austral de Chile. Real Jardín Botánico de Edimburgo. 187 pp.

Kruger, F.J., G.J. Breytenbach, I.A.W. Macdonald \& D.M. RichARDSON. 1989. The characteristics of invaded Mediterranean climate regions. Biological invasions. In: J.A. Drake, H.A. Mooney, F. di Castri, R.H. Groves, F.J. Krüger, M. Rejmanek \& M. Williamson (eds.), Biological Invasions: a Global Perspective, pp. 181-213. Wiley, Chichester, UK.

Lara, A. \& T.T. Veblen. 1993. Forest plantations in Chile: a successful model? In: A. Mather (ed.), Afforestation policies, planning and progress: 118-139. Belhaven Press, London, United Kingdom.

Ramírez, C., H Figueroa, R. Carrillo \& D. Contreras. 1984. Estudio Fitosociológico de los estratos inferiores en un Bosque de Pino (Valdivia, Chile). Revista Bosque (5)2: 65-81.
Richardson, D.M. \& P.J. BRown.1986. Invasion of mesic mountain fynbos by Pinus radiata. South African Journal of Botany 52: 529-536.

Richardson, D.M. \& S.I. Higgins. 1998. Pines as invaders in the southern hemisphere In: D.M. Richardson (ed.), Ecology and Biogeography of Pinus, pp. 450-473. Cambridge University Press, Cambridge.

Richardson, D.M., P. Pysek, M. Rejmánek, M.G. Barbour, F.D. PANETt \& C.T. West. 2000. Naturalization and invasion of alien plant: concepts and definitions. Diversity and Distributions 6: 93-107.

San Martín, J. \& C. Donoso. 1996. Estructura florística e impacto antrópico en el Bosque Maulino de Chile. En: J.J. Armesto, C. Villagrán \& M.T.K. Arroyo (eds.), Ecología de los bosques nativos de Chile. pp. 153-168. Editorial Universitaria, Santiago.

Recibido: 18.11 .10

Aceptado: 13.01.11 